\title{
Preliminary results using a P300 brain-computer interface speller: A possible interaction effect between presentation paradigm and set of stimuli
}

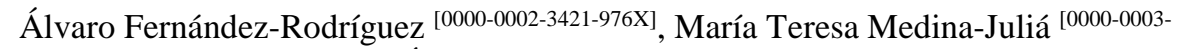

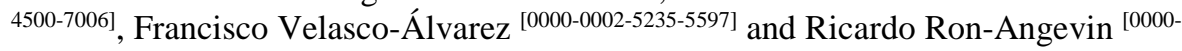 \\ 0001-8721-0585] \\ Departamento de Tecnología electrónica, Universidad de Málaga, 29071, Malaga, Spain \\ afernandezrguez@uma.es \\ maytemed@uma.es \\ fvelasco@dte.uma.es \\ rron@uma.es
}

\begin{abstract}
Several proposals to improve the performance controlling a P300based BCI speller have been studied using the standard row-column presentation (RCP) paradigm. However, this paradigm could not be suitable for those patients with lack of gaze control. To solve that, the rapid serial visual presentation (RSVP) paradigm, which presents the stimuli located in the same position, has been proposed in previous studies. Thus, the aim of the present work is to assess if a stimuli set of pictures that improves the performance in RCP, could also improve the performance in a RSVP paradigm. Six able-bodied participants have controlled four conditions in a calibration task: letters in RCP, pictures in RCP, letters in RSVP and pictures in RSVP. The results showed that pictures in RCP obtained the best accuracy and information transfer rate. The improvement effect given by pictures was greater in the RCP paradigm than in RSVP. Therefore, the improvements reached under RCP may not be directly transferred to the RSVP.
\end{abstract}

Keywords: brain-computer interface (BCI), P300, speller, stimuli, rapid serial visual presentation (RSVP).

\section{Introduction}

Several diseases may provoke deterioration in the motor abilities of affected patients. Some of these diseases, such as the amyotrophic lateral sclerosis (ALS), can cause the locked-in state, in which the patient has his whole body immobilized except for some ocular movements [1].

Numerous systems, that make use of the motor skills remaining in patients, have been developed to give those patients a communication channel. Some examples are push-buttons or eye-trackers [2]. However, more severe cases of the ALS disease lead to the so-called complete locked-in state, in which there is even no presence of gaze 
control $[3,4]$. In the case of these patients, the communication system cannot be supported by any kind of muscular movements, including eye-gaze. This is when braincomputer interfaces (BCI) come into play. BCI is a type of technology that uses the patient's brain signal to establish a channel of communication between him/her and the device to be controlled [5]. Due to its relatively low cost and adequate temporal resolution, the technology most widely used for recording the brain signal is the electroencephalography (EEG).

Verbal communication is one of the main needs for patients, to the point of being able to take important decisions as receiving life-sustaining treatments [6]. Therefore, it is not surprising that the virtual speller is the application most developed by researchers in the field of BCI. The first BCI speller was proposed in 1988 by Farwell and Donchin [7]. It consisted of a matrix of letters whose rows and columns were flashed at random - it is called row-column presentation (RCP) - while the user had to focus his attention on the target letter. Each time that flashes the row or column which contains the target letter, the user perceives its flash and consequently it is evoked an eventrelated potential (ERP), known as P300, on the user's brain signal. Specifically, the P300 potential is a positive deflection in the voltage of the EEG signal, which is generally registered from the parietal lobe of the cortex and around $300 \mathrm{~ms}$ after the presentation of an uncommon target stimulus [8].

The P300 potential when used in a speller is influenced by numerous parameters, such as the number of elements of the matrix, the stimuli presentation duration or the use of some stimuli over others (see [9] for a detailed review of BCI spellers). In relation to this last parameter, the presented stimuli, it has been shown how the use of symbols of different colors or complex figures, instead of simple letters, can increase performance [10-12]. However, other paradigms have gone beyond the flashing of the symbol to be selected, and have opted for the superposition of the letters with certain images that increase the amplitude of the P300. To this day, the stimulus with the best results are familiar faces $[13,14]$. However, a preliminary study carried out by the research group of the present study - the UMA BCI group - revealed the possibility that a set of varied different pictures (e.g., photographs of things, people or places) as flashing stimuli - a different one for each symbol - could improve the performance compared to the classical paradigm of letters [15].

It should not be forgotten that the main purpose of these applications is to offer an additional channel of communication to patients with severely impaired motor skills, including the ability of ocular mobility. Therefore, the presentation paradigm is a factor to consider in order to meet the aim of establishing a communication channel for those patients. Currently, the most used presentation paradigm for a speller is the RCP; however, this paradigm requires the user to focus on the target symbol. Consequently, it has been proved that the user's performance is drastically affected when the user cannot focus on the desired symbol $[16,17]$. To solve this problem, another kind of presentation paradigm that does not require ocular mobility has been shown. The most developed paradigm for this purpose is the rapid serial visual presentation (RSVP) with the symbols located in the same position (e.g., [17-21]).

Despite the fact that the most suitable presentation paradigm for patients could be the RSVP, most of proposals have been developed under the RCP paradigm. Thus, it 
should be questioned if the findings made in RCP can be applied in RSVP with the stimuli presented in the same position. For example, in RSVP, the stimuli could compete with each other as they all appear in the same position in the screen. While in the RCP paradigm, in the position of the desired symbol, only the stimulus of the corresponding symbol appears. This implicates that the positive deflection of the EEG caused by the non-target stimuli can only occur due to the peripheral vision in RCP, but in RSVP it occurs due to the central vision. On the other hand, the positive deflection of the EEG caused by the target stimuli occurs due to the central vision in both paradigms. Therefore, those stimuli that increase the amplitude of the P300 signal could produce a clear improvement in RCP performance, but not in RSVP.

In short, the aim of the present study is to test two stimuli sets under two different presentation paradigms to assess the differences between conditions. Our hypothesis is that in RCP the benefit of pictures versus letters will be much greater than in the RSVP paradigm.

\section{Method}

\subsection{Participants}

The experiment involved six able-bodied participants (aged $27.17 \pm 6.04$, 3 females) who had normal or corrected-to-normal vision. Three subjects had previous experience controlling BCI systems and the other three did not. The study was approved by the Ethics Committee of the University of Malaga and met the ethical standards of the Helsinki Declaration. According to self-reports, none of the participants had any history of neurological or psychiatric illness or were taking any medication regularly.

\subsection{Data acquisition and signal processing}

The EEG was recorded at a sample rate of $250 \mathrm{~Hz}$ using the electrode positions: $\mathrm{Fz}, \mathrm{Cz}$, $\mathrm{Pz}, \mathrm{Oz}, \mathrm{P} 3, \mathrm{P} 4, \mathrm{PO} 7$ and PO8, according to the 10/20 international system. All channels were referenced to TP8 and grounded to position AFz. Signals were amplified by an acti-CHamp amplifier (Brain Products GmbH, Munich, Germany). Neither online nor offline artifact detection techniques were employed. All aspects of EEG data collection and processing were controlled by the BCI2000 system [22]. A Stepwise Linear Discriminant Analysis (SWLDA) of the EEG data was performed to obtain the weights for the P300 classifier and calculate the accuracy. The software used to design the interface was developed by the UMA-BCI group from the University of Malaga. This software is named UMA-BCI Speller, and serves as a friendly front-end of BCI2000.

\subsection{The spelling conditions}

The present work employed four conditions, based on the combination of two paradigms (RCP and RSVP) and two sets of stimuli (letters versus pictures) (figure 1). The 
only difference between the compared conditions was the employed presentation paradigm and flashing stimuli for each condition. Thus, the four presented conditions were: i) letters in RCP (L-RCP), ii) pictures in RCP (P-RCP), iii) letters in RSVP (L-RSVP), and iv) pictures in RSVP (P-RSVP) (figure 1). The font used for letters was arial bold in capital letters. On the one hand, the RCP conditions $(2 \times 3$ matrix size) were initially based on the previously mentioned paradigm of [7]. On the other hand, for the RSVP, only the flashing stimuli were presented, placed in the middle of the screen. Letters had a size around $3 \mathrm{~cm} \times 3.5 \mathrm{~cm}$, depending on the letter. Regarding the pictures, their size was equal to $4.7 \mathrm{~cm} \times 3.5 \mathrm{~cm}(187 \mathrm{px} \times 140 \mathrm{px})$. The conditions were displayed on a 15.6-in $(39.6 \mathrm{~cm})$ screen at a distance of $60 \mathrm{~cm}$ and at a refresh rate of $60 \mathrm{~Hz}$. For all conditions, a stimulus onset asynchrony (SOA) of $288 \mathrm{~ms}$ and an inter-stimulus interval (ISI) of 96 ms were used, so each stimulus was presented for $192 \mathrm{~ms}$. A 3968 ms pause was established between each selection.

\section{L-RCP}

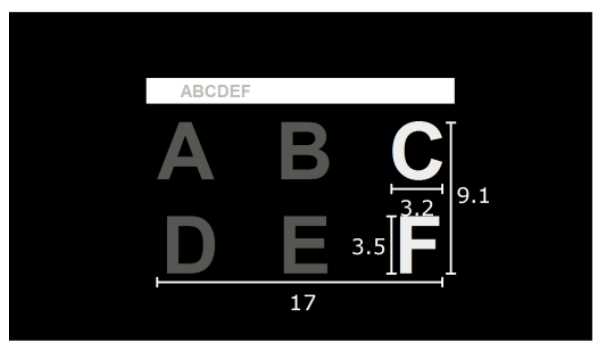

L-RSVP

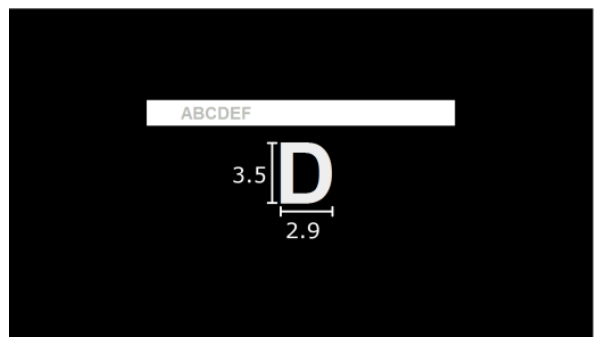

\section{P-RCP}

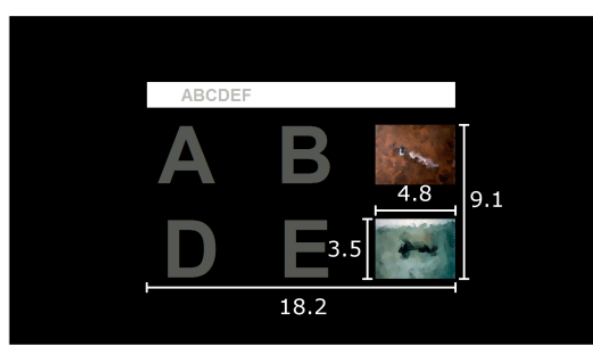

P-RSVP

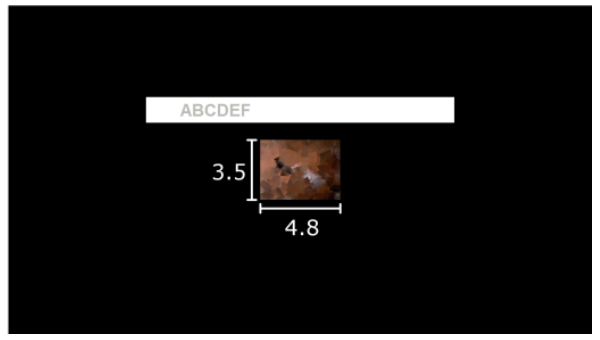

Fig. 1. Size metrics (cm) for all conditions: letters in row-column presentation (L-RCP), pictures in row-column presentation (P-RCP), letters in rapid serial visual presentation (L-RSVP), and pictures in rapid serial visual presentation (P-RSVP). Due to copyright reasons, the condition with pictures has been pixelated.

Regarding the pictures, they were obtained from the International Affective Picture System (IAPS) [23]. Those images with the lowest score in the level of excitation were chosen, and also that filled the proportion of the aforementioned size (i.e., those pictures that filled all the space and did not have black paddings). The specific selected pictures according to the IAPS codification for the matrix in major-row order were: 7175,7010 , 7004, 7031, 7020 and 7110. 


\subsection{Procedure}

The experiment was carried out in an isolated room where only the participant was present at the time he/she was performing the task in order to concentrate on it without external distractions. It consisted of only one exercise: a calibration task to adapt the system to the user. There was no writing task in which the user actually controlled the interface. Consequently, the study was performed in one session.

An intrasubject, also called repeated measures, design was used, and so all the users went through all the experimental conditions.

The experiment was carried out in 16 blocks of six selections, so each condition was performed in four blocks (i.e., 24 selections per condition). The symbol selection order for each block was A, B, C, D, E and F. The conditions were presented pseudo-randomly to prevent any unwanted effect, such as learning or fatigue, and all conditions were equally distributed. There was a short break between blocks (variable at the request of the user). The number of sequences per run (when the user is selecting one symbol) was equal to 6 , so each symbol flashed 12 times in RCP (6 flashes per row and 6 flashes per column) and 6 times in RSVP. The participant was asked to count these flashes in order to keep his/her attention on the task. The writing time for each symbol was $8.54 \mathrm{~s}$ in RCP and $10.27 \mathrm{~s}$ in RSVP.

\section{$2.5 \quad$ Evaluation}

Two parameters were used to evaluate the effect of the presentation paradigm and stimuli type on the performance: i) the accuracy of the system classifying the selections (i.e., the number of correctly predicted selections divided by the total number of predicted selections) and ii) the information transfer rate (ITR, bits/min) based on the formula presented in [24]. It should be advised that the pause between selections is not considered to calculate the ITR. In addition, the ERP signal was analyzed in order to observe how the brain signal activity is affected by the application of different sets of stimuli and paradigm. Specifically, the dependent variable employed was the amplitude difference in absolute value between target and no target stimuli signals $(\mu V)$ from 200 to $600 \mathrm{~ms}$.

Due to the small sample size, non-parametric analyses were carried out. Initially, a Freidman's test was performed to find differences between conditions. Then, multiple Wilcoxon signed-rank tests were carried out in order to find those specific differences between conditions. Due to the preliminary nature of the present study, no correction method was applied for multiple comparisons. The obtained conclusions should be considered carefully, so more tests will be necessary to replicate the results and avoid type I error. 


\section{Results and discussion}

\subsection{Accuracy}

Figure 2 visually shows how the accuracy of the different conditions become similar as the sequences increase. In general, all conditions offered good results in accuracy. However, it should be noted that, despite there being no significant differences, the PRCP condition obtained 100\% accuracy for all participants from sequence 2 .

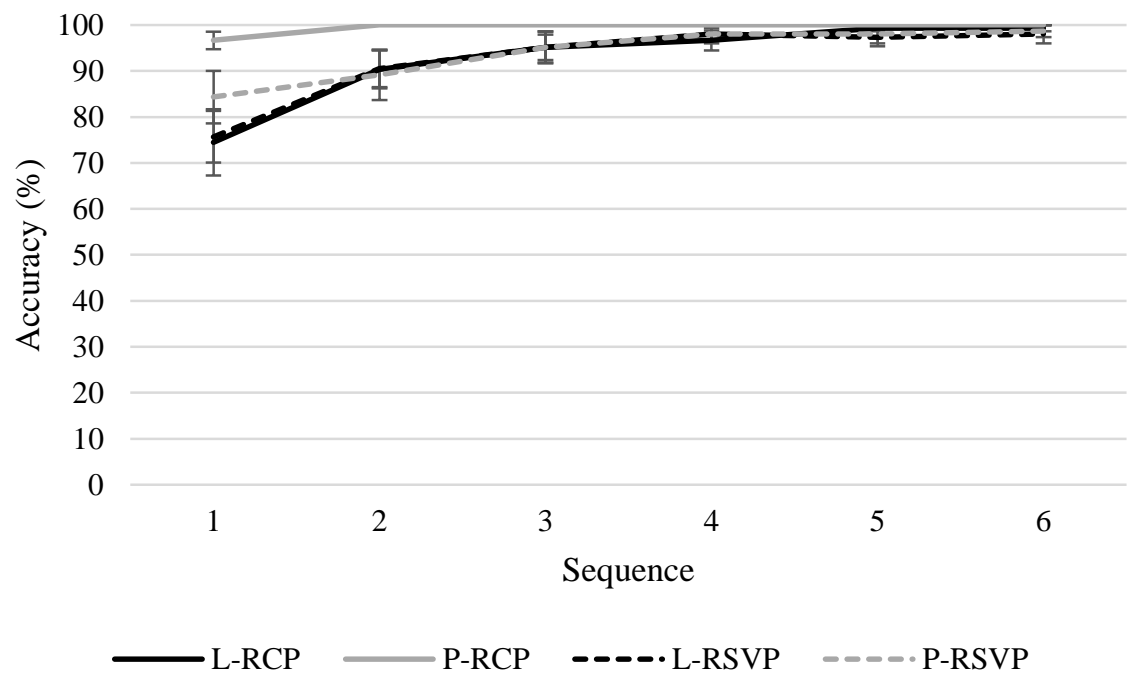

Fig. 2. Accuracy (mean \pm standard error) of the different P300-speller conditions as a function of the number of sequences.

According to the Friedman's tests, significant differences between conditions were found only in the first sequence $\left(\chi^{2}(3)=12.273 ; p=.007\right)$. The specific differences were obtained between P-RCP versus L-RCP $(Z=2.795 ; p=.005)$ and versus L-RSVP $(Z=-2.795 ; p=.005)$, i.e., between the P-RCP and the two conditions with letters. Meanwhile, the P-RSVP did not offer any significant difference, so the effect of pictures in RSVP is not as strong as in RCP. This could guide us in future studies to look for an interaction effect between the presentation paradigm and the stimulus employed.

Averaging the results of the picture conditions (P-RCP and P-RSVP) versus those of letters (L-RCP and L-RSVP), it was obtained that in sequence 1 the picture conditions obtained a better accuracy $(Z=2.201 ; p=.028)$. However, this effect was already lost from sequence 2 . On the other hand, in reference to the averaging of conditions under RCP (L-RCP and P-RCP) and RSVP (L-RSVP and P-RSVP) paradigms, no significant differences between presentation paradigms were found, in any sequence. Nevertheless, in spite of not having found a significant effect between RCP and RSVP, in the visual analysis of figure 2 a trend can be observed in the first sequence according to which the benefit of applying pictures is greater in the RCP paradigm than in RSVP. 


\subsection{Information transfer rate (ITR)}

Figure 3 shows the change of the ITR as the sequences increased. Due to the suitable results in accuracy, all conditions achieved a proper ITR. The superiority of the P-RCP paradigm was shown again, which in this case is further increased due to the trend of the RCP paradigms to offer a higher ITR. This superiority of the RCP paradigms in terms of ITR is observed in the fact that the L-RCP paradigm, although having a similar accuracy to the RSVP paradigms, obtains a higher ITR.

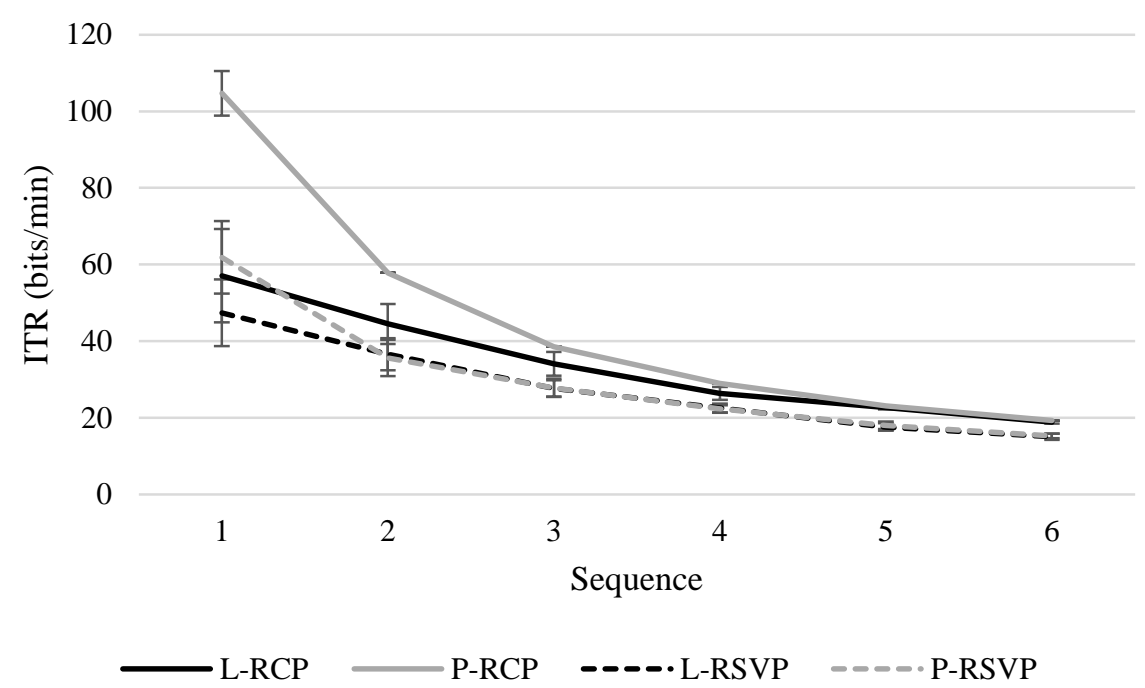

Fig. 3. Information transfer rate (mean \pm standard error) of the different P300-speller conditions as a function of the number of sequences.

According to the Friedman's test, significant differences were found between conditions for every sequence: sequence $1\left(\chi^{2}(3)=12.966 ; p=.005\right)$, sequence $2\left(\chi^{2}(3)=\right.$ $10.286 ; p=.016)$, sequence $3\left(\chi^{2}(3)=9.34 ; p=.025\right)$, sequence $4\left(\chi^{2}(3)=9.462 ; p=\right.$ $.024)$, sequence $5\left(\chi^{2}(3)=17.4 ; p=.001\right)$ and sequence $6\left(\chi^{2}(3)=17.4 ; p=.001\right)$. Table 1 shows the multiple comparison test between conditions. In the first sequence, significant differences were obtained only for P-RCP compared to the rest of conditions (L-RCP, L-RSVP and P-RSVP). These results showed that the effect of improvement produced by pictures was only observed in RCP, while in RSVP the stimuli used do not influence significant differences in ITR. From sequence 2 both presentation paradigms are dissociated, leading to a complete dissociation in sequences 5 and 6 (figure 3).

Table 1. Average information transfer rate (ITR; mean \pm standard error) for each condition and sequence during the calibration task.

\begin{tabular}{ccccc}
\hline \multirow{2}{*}{ Sequence } & \multicolumn{4}{c}{ Condition } \\
\cline { 2 - 5 } & L-RCP (1) & P-RCP (2) & L-RSVP (3) & P-RSVP (4) \\
\hline 1 & $57.06 \pm 12.18^{2}$ & $104.67 \pm 5.84^{1,3,4}$ & $47.4 \pm 8.74^{2}$ & $61.84 \pm 9.45^{2}$
\end{tabular}




$\begin{array}{lcccc}2 & 44.47 \pm 5.27 & 57.83 \pm 0^{3,4} & 36.6 \pm 4.18^{2} & 35.64 \pm 4.74^{2} \\ 3 & 34.04 \pm 3.11 & 38.56 \pm 0^{3,4} & 27.63 \pm 2.14^{2} & 27.79 \pm 2.38^{2} \\ 4 & 26.32 \pm 1.67 & 28.92 \pm 0^{3,4} & 22.51 \pm 1.24^{2} & 22.33 \pm 0.94^{2} \\ 5 & 22.64 \pm 0.5^{3,4} & 23.13 \pm 0^{3,4} & 17.6 \pm 0.99^{1,2} & 18.01 \pm 0.99^{1,2} \\ 6 & 18.86 \pm 0.41^{3,4} & 19.28 \pm 0^{3,4} & 15.01 \pm 0.82^{1,2} & 15.23 \pm 0.6^{1,2}\end{array}$

Note: letters in row-column presentation (L-RCP), pictures in row-column presentation (P-RCP), letters in rapid serial visual presentation (L-RSVP), and pictures in rapid serial visual presentation (P-RSVP). Significant differences between conditions $(p<.05)$ are denoted with a superindex to show which speller average was different to (1 for L-RCP, 2 for P-RCP, 3 for L-RSVP, and 4 for P-RSVP).

In reference to the average of the conditions relative to the stimuli of letters and pictures, the picture conditions only offered a higher ITR versus the letters conditions in the first sequence $(Z=2.201 ; p=.028)$. However, the effect of the presentation paradigm was found in all sequences: sequence $1(Z=-1.992, p=.046)$, sequence $2(Z=$ $-2.201 ; p=.028)$, sequence $3(Z=-2.201 ; p=.028)$, sequence $4(Z=-2.207 ; p=.027)$, sequence $5(Z=-2.226 ; p=.026)$ and sequence $6(Z=-2.264 ; p=.024)$. These results corroborate what was previously declared in [9], according to which the paradigm RSVP trends to offer a lower ITR than RCP. In this case, this difference is especially notable since the accuracy obtained has been suitable, as most of participants needed just a few sequences to reach $100 \%$ accuracy with the four conditions. The ITR has been exclusively dependent on the presentation speed of the stimuli. Perhaps, this ceiling effect for accuracy should be avoided by applying even faster presentation times.

\subsection{Event-related potential (ERP) waveform}

Figure 4 shows the average amplitude of the EEG signal for each condition, electrode and stimuli (target or non-target). However, as it was previously explained, the dependent variable used for next analysis was the amplitude difference in absolute value between target and non-target stimuli $(\mu \mathrm{V})$. According to the Friedman's test, significant differences were obtained in amplitude differences for the next electrodes: $\mathrm{Cz}\left(\chi^{2}(3)=\right.$ 8.6; $p=.035), \mathrm{Pz}\left(\chi^{2}(3)=11 ; p=.012\right), \mathrm{Oz}\left(\chi^{2}(3)=10 ; p=.019\right)$ and $\mathrm{P} 4\left(\chi^{2}(3)=15\right.$; $p=.002$ ). Electrodes Fz, P3, PO7 and PO8 did not offer any significant differences. Table 2 shows the multiple comparison test between conditions. 


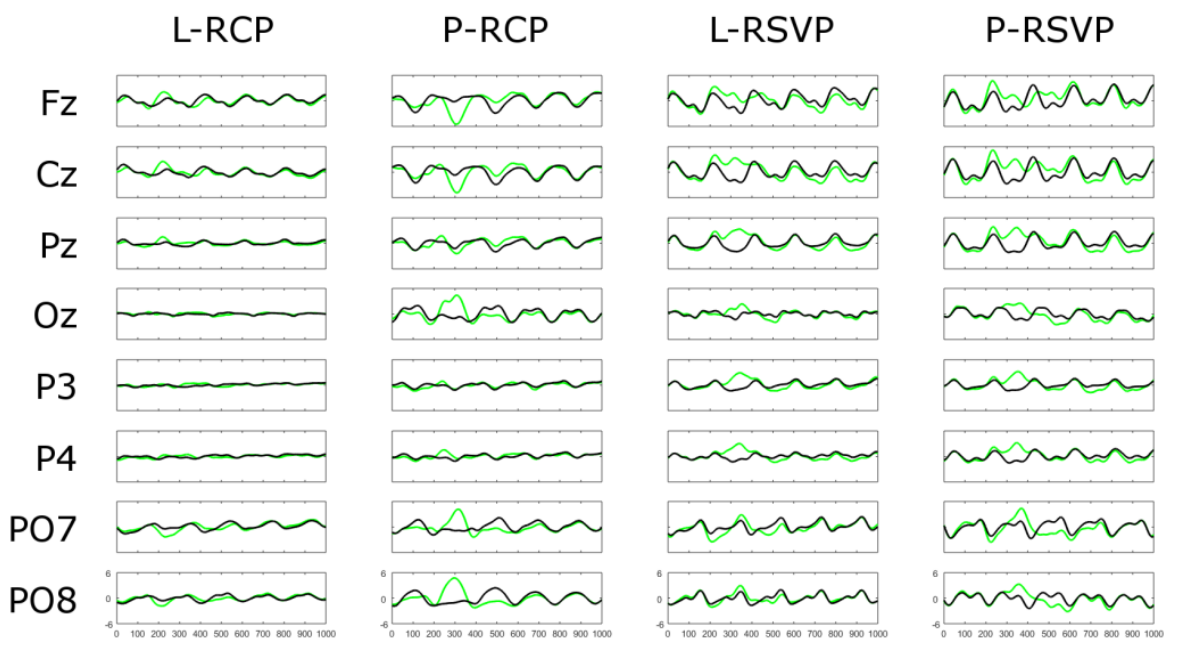

Fig. 4. Grand average event-related (ERP) potential waveforms $(\mu \mathrm{V})$ for target - green - and non-target stimuli - black - for the eight used electrodes and spellers (letters in row-column presentation (L-RCP), pictures in row-column presentation (P-RCP), letters in rapid serial visual presentation (L-RSVP), and pictures in rapid serial visual presentation (P-RSVP)).

Table 2. Average amplitude differences in absolute value ( $\mu \mathrm{V}$; mean \pm standard error) for each condition and electrode position during the calibration task.

\begin{tabular}{ccccc}
\hline \multirow{2}{*}{ Electrode } & \multicolumn{4}{c}{ Condition } \\
\cline { 2 - 5 } & L-RCP (1) & P-RCP (2) & L-RSVP (3) & P-RSVP (4) \\
\hline Fz & $1.39 \pm 0.18$ & $2.49 \pm 0.3$ & $1.93 \pm 0.33$ & $2.12 \pm 0.22$ \\
Cz & $1.32 \pm 0.17^{2,3,4}$ & $2.03 \pm 0.29^{1}$ & $2.67 \pm 0.41^{1}$ & $2.6 \pm 0.39^{1}$ \\
Pz & $0.9 \pm 0.1^{3,4}$ & $1.27 \pm 0.29^{4}$ & $2.38 \pm 0.37^{1}$ & $2.45 \pm 0.31^{1,2}$ \\
Oz & $0.71 \pm 0.14^{2}$ & $2.56 \pm 0.42^{1}$ & $1.23 \pm 0.26$ & $1.51 \pm 0.41$ \\
P3 & $0.87 \pm 0.14$ & $0.88 \pm 0.11^{1}$ & $1.82 \pm 0.41$ & $1.74 \pm 0.31$ \\
P4 & $0.56 \pm 0.07^{3,4}$ & $0.96 \pm 0.09^{4}$ & $1.65 \pm 0.27^{1}$ & $1.71 \pm 0.23^{1,2}$ \\
PO7 & $1.37 \pm 0.38$ & $2.31 \pm 0.4$ & $1.76 \pm 0.38$ & $1.78 \pm 0.49$ \\
PO8 & $1.02 \pm 0.21$ & $2.58 \pm 0.54$ & $1.03 \pm 0.17$ & $1.27 \pm 0.32$ \\
\hline
\end{tabular}

Note: letters in row-column presentation (L-RCP), pictures in row-column presentation (P-RCP), letters in rapid serial visual presentation (L-RSVP), and pictures in rapid serial visual presentation (P-RSVP). Significant differences between conditions $(p<.05)$ are denoted with a superindex to show which speller average was different to (1 for L-RCP, 2 for P-RCP, 3 for L-RSVP, and 4 for P-RSVP).

As for the previous variables, it has been decided to perform the average amplitude difference in relation to the stimulus and presentation paradigm factors. On the one hand, for the averaging of the stimuli, it was obtained that the picture conditions presented a higher amplitude difference for the following positions: $\mathrm{Oz}(Z=2.201, p=$ $.028), \mathrm{P} 4(Z=2.201, p=.028), \mathrm{PO} 7(Z=1.992, p=.046)$ and PO8 $(Z=1.992, p=$ 
.046). On the other hand, contrary to what was expected, the different Wilcoxon signedrank test did not offer significant differences by averaging the conditions according to the paradigm of the stimuli presentation.

An interesting point of figure 4 is that in the channels $\mathrm{Fz}$ and $\mathrm{Cz}$ for the conditions L-RCP, L-RSVP and P-RSVP, the peak of amplitude difference between target and non-target (around 200-400 ms) showed a higher amplitude for the target. However, for the P-RCP condition, the opposite effect is observed: clearly smaller amplitude of the target versus the non-target. This effect has not been previously reported in other work and, thus, it should be specifically studied in future works.

\section{Conclusions}

The general trend of the present work has been to show the P-RCP as the condition with the best performance. Otherwise, the other conditions were fairly even. While differences in performance between the RCP conditions have been found, the conditions under RSVP paradigm always obtained non-significant results between them. In fact, L-RSVP and P-RSVP offered similar results from the second sequence.

Despite the fact that the P-RCP condition has shown the best performance, it must be remembered that the study has been carried out under an overt attention condition, i.e., users could move their eyes unrestrictedly. As we said in the introduction, the ocular mobility is a capacity that is not present in severely affected patients.

As future proposals related to this work, it should be considered to replicate these results with a larger sample size in order to obtain more reliable results and be able to perform the appropriate statistical analyses. Likewise, it would be convenient to test these results in an online writing session, since this would be the real experience of controlling a BCI speller. On the other hand, it would also be interesting to employ a broader approach when evaluating the different conditions, such as the usability construct defined by [25].

In short, the present preliminary study has found that the effect of the pictures depends on the presentation paradigm used. These results show that the contributions made in RCP - the paradigm most used today - should not be directly considered as valid in RSVP without testing them.

Acknowledgements. This work was partially supported by the Spanish Ministry of Economy and Competitiveness through the projects LICOM (DPI2015-67064-R), by the European Regional Development Fund (ERDF) and by the University of Malaga. Moreover, the authors would like to thank all participants for their cooperation.

\section{References}

1. Patterson, J.R., Grabois, M.: Locked-in syndrome: A review of 139 cases. Stroke. 17, 758-764 (1986).

2. Pal, S., Mangal, N.K., Khosla, A.: Development of assistive application for patients with 
communication disability. IEEE Int. Conf. Innov. Green Energy Healthc. Technol. 2017, IGEHT 2017. 1-4 (2017).

3. Bauer, G., Gerstenbrand, F., Rumpl, E.: Varieties of the locked-in syndrome. J. Neurol. 221, 77-91 (1979).

4. Murguialday, A.R., Hill, J., Bensch, M., Martens, S., Halder, S., Nijboer, F., Schoelkopf, B., Birbaumer, N., Gharabaghi, A.: Transition from the locked in to the completely locked-in state: A physiological analysis. Clin. Neurophysiol. 122, 925-933 (2011).

5. Birbaumer, N.: Breaking the silence: Brain-computer interfaces (BCI) for communication and motor control. Psychophysiology. 43, 517-532 (2006).

6. Lemoignan, J., Ells, C.: Amyotrophic lateral sclerosis and assisted ventilation: How patients decide. Palliat. Support. Care. 8, 207-213 (2010).

7. Farwell, L.A., Donchin, E.: Talking off the top of your head: toward a mental prosthesis utilizing event-related brain potentials. Electroencephalogr. Clin. Neurophysiol. 70, 510-523 (1988).

8. Nicolas-Alonso, L.F., Gomez-Gil, J.: Brain computer interfaces, a review. Sensors. 12, 1211-1279 (2012).

9. Rezeika, A., Benda, M., Stawicki, P., Gembler, F., Saboor, A., Volosyak, I.: Braincomputer interface spellers: A review. Brain Sci. 8, (2018).

10. Ryan, D.B., Townsend, G., Gates, N.A., Colwell, K., Sellers, E.W.: Evaluating braincomputer interface performance using color in the P300 checkerboard speller. Clin. Neurophysiol. 128, 2050-2057 (2017).

11. Ryan, D.B., Colwell, K.A., Throckmorton, C.S., Collins, L.M., Caves, K., Sellers, E.W., Throckmorton, S., Collins, L.M., Sellers, E.W., Throckmorton, C.S., Collins, L.M., Caves, K., Sellers, E.W.: Evaluating Brain-Computer Interface Performance in an ALS Population: Checkerboard and Color Paradigms. Clin. EEG Neurosci. 49, 114-121 (2018).

12. Ma, Z., Qiu, T.: Performance improvement of ERP-based brain-computer interface via varied geometric patterns. Med. Biol. Eng. Comput. 55, 2245-2256 (2017).

13. Kaufmann, T., Schulz, S.M., Grünzinger, C., Kübler, A.: Flashing characters with famous faces improves ERP-based brain-computer interface performance, http://stacks.iop.org/1741-

2552/8/i=5/a=056016?key=crossref.04cfedc9b1db574d6b4a9c9ee9a759f9, (2011).

14. Li, Q., Liu, S., Li, J., Bai, O.: Use of a green familiar faces paradigm improves P300speller brain-computer interface performance. PLoS One. 10, 1-15 (2015).

15. Fernández-Rodríguez, Á., Velasco-Álvarez, F., Ron-Angevin, R.: Evaluation of a P300 Brain-Computer Interface Using Different Sets of Flashing Stimuli. In: Ron-angevin, R. (ed.) BRAININFO 2018: The Third International Conference on Neuroscience and Cognitive Brain Information. pp. 1-4. IARIA, Venice (2018).

16. Brunner, P., Joshi, S., Briskin, S., Wolpaw, J.R., Bischof, H., Schalk, G.: Does the "P300” speller depend on eye gaze? J. Neural Eng. 7, 056013 (2010).

17. Treder, M.S., Blankertz, B.: (C)overt attention and visual speller design in an ERP-based brain-computer interface. Behav. Brain Funct. (2010).

18. Acqualagna, L., Treder, M.S., Schreuder, M., Blankertz, B.: A novel brain-computer interface based on the rapid serial visual presentation paradigm. In: 2010 Annual International Conference of the IEEE Engineering in Medicine and Biology Society, 
EMBC'10 (2010).

19. Acqualagna, L., Treder, M.S., Blankertz, B.: Chroma Speller: Isotropic visual stimuli for truly gaze-independent spelling. Int. IEEE/EMBS Conf. Neural Eng. NER. 10411044 (2013).

20. Sato, H., Washizawa, Y.: An N100-P300 Spelling Brain-Computer Interface with Detection of Intentional Control. Computers. 5, 31 (2016).

21. Aricò, P., Aloise, F., Schettini, F., Riccio, A., Salinari, S.: GeoSpell : an alternative P300-based speller interface towards no eye gaze required. Int. J. Bioelectromagn. 13, 152-153 (2011).

22. Schalk, G., McFarland, D.J., Hinterberger, T., Birbaumer, N., Wolpaw, J.R.: BCI2000: A general-purpose brain-computer interface (BCI) system, (2004).

23. Lang, P. J., Bradley, M. M., \& Cuthbert, B.N.: International affective picture system (IAPS): Affective ratings of pictures and instruction manual. Technical Report A-8. University of Florida, Gainesville, FL. (2008).

24. Wolpaw, J.R., Ramoser, H., McFarland, D.J., Pfurtscheller, G.: EEG-based communication: Improved accuracy by response verification. IEEE Trans. Rehabil. Eng. 6, 326-333 (1998).

25. ISO: ISO9241-11: Ergonomic requirements for office work with visual display terminals (VDTs). Part11 Guid. usability. 11, 22 (1994). 